\title{
Modeling and Simulation of Three Phase PWM Rectifier Based on One-cycle Control
}

\author{
Zhang Shutuan \\ Department of Control Engineering \\ Naval Aeronautical and Astronautical University \\ Yantai, China, 264001
}

\author{
Pi Zhijun, Xiao zhicai \\ Department of Control Engineering \\ Naval Aeronautical and Astronautical University \\ Yantai, China, 264001
}

\begin{abstract}
In this paper, the three phase rectifier based on onecycle control technique is introduced. The operation principle of the one-cycle control is given, and the simulation models based on the saber software is constructed. The simulation results show that the input current of the rectifier is identical with its corresponding input voltage, and the rectifier can realize high power factor by adopting the one-cycle control technique, and achieve the design requirement, and the three phase rectifier based on one-cycle control is very reliable and stable method.
\end{abstract}

Keywords-Rectifier; One-cycle control technique; Power factor; Simulation

\section{INTRODUCTION}

Nowadays, with the development of modern power electronics technologies, the application of the traditional transformer rectifier and equipments with nonlinear loads have widely used in the aircraft. All of them results in serious harmonic problems in the utility grid and cause great impact for the aircraft power supply systems and power quality. In order to reduce the harmonic and increase the power factor, there are two basic methods usually used. The first method is compensating the harmonics and reactive power by installing the compensation device; the second method is improving the power electronic devices. The improved device can not produce reactive power, or as needed to control their power factor. Compare the method of improving the power electronic devices with the first method, the second method is more active and effective way[1-3]. In this way, we can design a new generation of high performance rectifier, which can't produce harmonics and realize unit power factor. So, the implement of new high power factor rectifier become a research hotspot.

One-cycle control technique was used to the rectifiers in aircraft electric power system in paper [1]. The one-cycle control technique applied to three-phase rectifier can achieve low current distortion and high power factor. This control method can not use the multiplier, thus reduce the complexity of the control system, and has some characters such as fast dynamic response, constant switching frequency, easy to implement, etc.

This paper will be structured as follows: In Section II, we will introduce operation principle of the one-cycle control. The control circuit design of the rectifier used in the aircraft is introduced in Section III; the system simulation based on saber software is given in section IV; In Section V, some simulation results of the rectifier are given.

\section{OPERATION PRINCIPLE[1]}

The three-phase three switch boost rectifier circuit diagram is shown in Fig.1. In order to analyze, the threephase voltage waveform in each cycle is divided into six intervals. According to the PWM control technology principle, the three-phase rectifier can realize unity power factor rectifier by controlling the work of two switches within each $60^{\circ}$ interval. The dual-boost topology is shown in Fig2. From the Fig.2, we can see that the two switches are driven by the same driver block; but only one switch can operate during each half cycle. In Fig.2, Tp or $\mathrm{Tn}$ is the switch in different intervals, $\mathrm{Vp}$ or $\mathrm{Vn}$ is the equivalent voltage in different intervals, and $\mathrm{Lp}, \mathrm{Ln}$ is the inductance in different intervals.

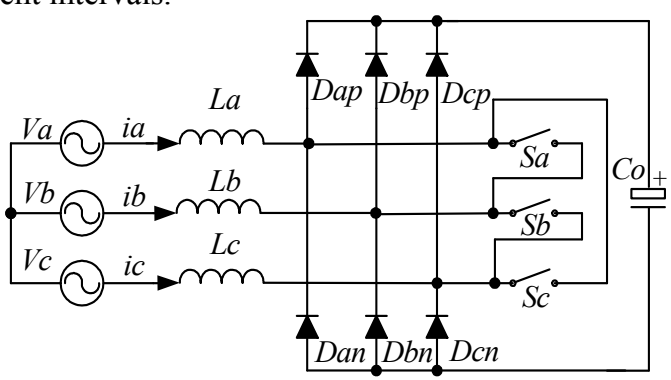

Figure 1. three-phase boost rectifier circuit diagram

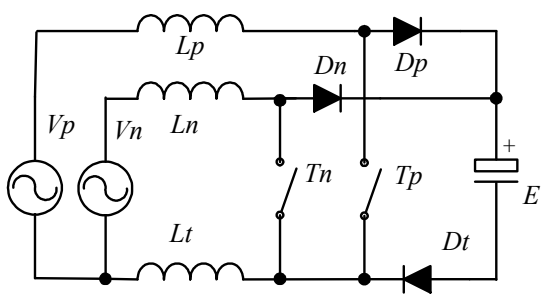

Figure 2. The dual-boost topology circuit

In the interval $(0 \sim 60)$, the equivalent voltage $V_{p}$ is either $V_{a}-V_{b}$ or $V_{c}-V_{b}$, the equivalent voltage $V_{p}$ in the other interval are similar. There are four possible switches state for the dual-boost topology circuit. For example, when 
the switch $T_{p}$ is turn on and the switch $T_{n}$ is turn off, the voltage of the three phase inductor is analyzed. The equation is as follows:

$$
\left\{\begin{array}{l}
V_{p}=L \cdot \frac{d i_{L p}}{d t}+L \cdot \frac{d i_{L t}}{d t} \\
V_{n}-E=L \cdot \frac{d i_{L t}}{d t}+L \cdot \frac{d i_{L n}}{d t} \\
i_{L t}=i_{L p}+i_{L n}
\end{array}\right.
$$

Where, the value $\mathrm{E}$ is the output voltage of DC side. The equation (1) can obtain equation (2) by calculating.

$$
\left\{\begin{array}{l}
V_{p}=2 L \cdot \frac{d i_{L p}}{d t}+L \cdot \frac{d i_{L n}}{d t} \\
V_{n}-E=L \cdot \frac{d i_{L p}}{d t}+2 L \cdot \frac{d i_{L n}}{d t}
\end{array}\right.
$$

According to equation (2), we can have:

$$
\left\{\begin{array}{l}
\frac{2 V_{p}-V_{n}+E}{3}=L \cdot \frac{d i_{L p}}{d t}=V_{L p} \\
\frac{2\left(V_{n}-E\right)-V_{p}}{3}=L \cdot \frac{d i_{L n}}{d t}=V_{L n} \\
\frac{V_{p}+V_{n}-E}{3}=L \cdot \frac{d i_{L t}}{d t}=V_{L t}
\end{array}\right.
$$

Similarly, the other switch status can be analyzed by adopting the similar analysis methods. We can obtain

$$
\left[\begin{array}{c}
V_{p}^{*} \\
V_{n}^{*} \\
V_{t}^{*}
\end{array}\right]=\left[\begin{array}{cc}
\frac{2}{3} & -\frac{1}{3} \\
-\frac{1}{3} & \frac{2}{3} \\
\frac{1}{3} & \frac{1}{3}
\end{array}\right] \cdot\left[\begin{array}{c}
V_{p} \\
V_{n}
\end{array}\right]
$$

Suppose, the switching frequency used in the rectifier is much higher than the frequency of the power system, then the average value can be looked as zero. For a balanced three-phase power system, the equation (5) can be given:

$$
\left\{\begin{array}{l}
V_{p}{ }^{*} \cdot d_{n}+\left(V_{p}^{*}+\frac{1}{3} \cdot E\right) \cdot\left(d_{p}-d_{n}\right)+\left(V_{p}^{*}-\frac{1}{3} \cdot E\right) \cdot\left(1-d_{p}\right)=0 \\
V_{n}{ }^{*} \cdot d_{n}+\left(V_{n}{ }^{*}-\frac{2}{3} \cdot E\right) \cdot\left(d_{p}-d_{n}\right)+\left(V_{n}{ }^{*}-\frac{1}{3} \cdot E\right) \cdot\left(1-d_{p}\right)=0 \\
V_{t}^{*} \cdot d_{n}+\left(V_{t}^{*}-\frac{1}{3} \cdot E\right) \cdot\left(d_{p}-d_{n}\right)+\left(V_{t}^{*}-\frac{2}{3} \cdot E\right) \cdot\left(1-d_{p}\right)=0
\end{array}\right.
$$

Because the power system is balanced system, so the equation (6) can be obtained:

$$
V_{p}^{*}+V_{n}^{*}-V_{t}^{*}=0
$$

According to the equation (5) and equation (6), the equation (7) can be given

$$
\left[\begin{array}{l}
1-d_{p} \\
1-d_{n}
\end{array}\right]=\left[\begin{array}{ll}
2 & 1 \\
1 & 2
\end{array}\right] \cdot\left[\begin{array}{c}
V_{p}^{*} / E \\
V_{n}^{*} / E
\end{array}\right]
$$

The equation (7) can be used in the other intervals.

\section{Control Circuit Design}

The control circuit of the three phase rectifier based on one cycle control technique is made up of the selection circuit, the input multiplexer circuit, the one cycle control circuit, the filter circuit and the output logic circuit. The core circuit of the control circuit of the three phase rectifier is divided into the adder circuit, the comparison circuit, and the integrator circuit, and so on. The adder circuit mainly realized the sum of the equations; the comparison circuit is used to realize the comparison of the data; the integrator circuit includes an integrator with reset, mainly used to produce the comparison signals. These equations can be realized by one-cycle control circuit of the three phase rectifier is shown in Fig.3.

The principle diagram of the core control circuit of the three phase rectifier is shown in Fig.3. In the control circuit, the absolute values of the current signals came from the three phase rectifier firstly be transmitted into the voltage signals by using the input multiplex circuit, and then be sent into the filter circuit; the signals from the filter circuit can be calculated according to those equations, and compared with the signals came from the integrator circuit; at last, the control signals will be export to the drive circuit after the output logic circuit [4].

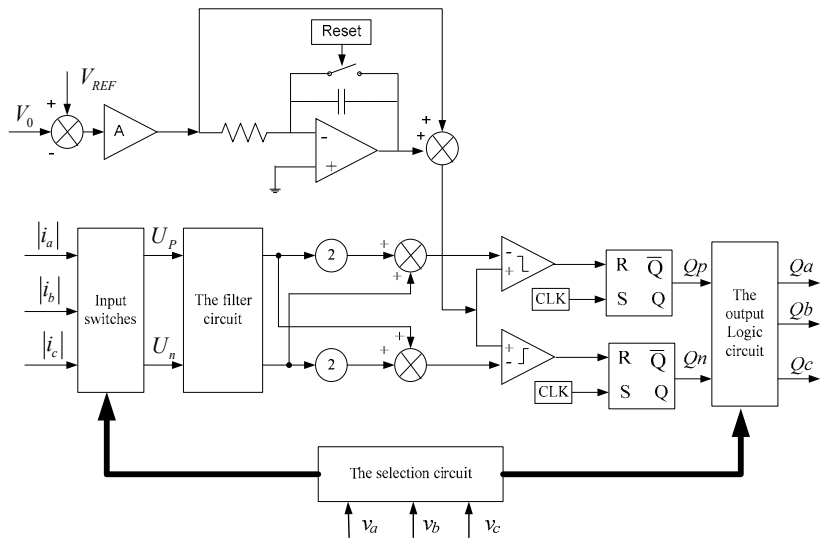

Figure 3. The principle diagram of one cycle control 


\section{System Simulation Based On SABer}

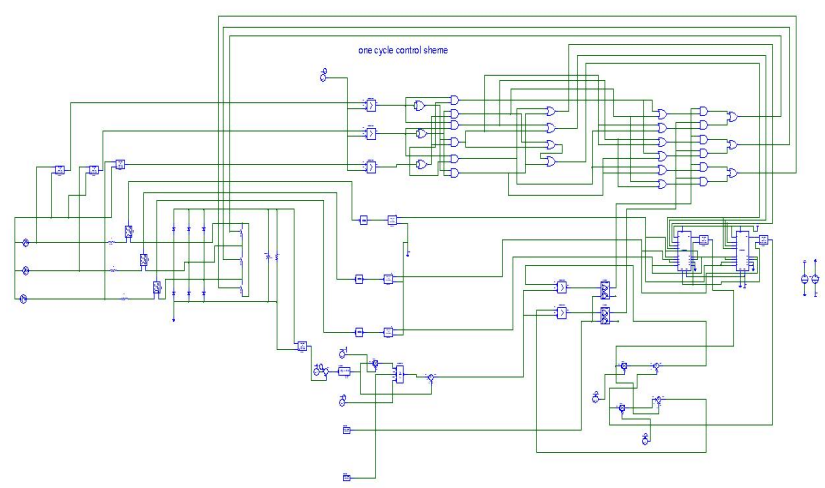

Figure 4. Simulation circuit of the rectifier

In order to verify the analysis of the rectifier, the simulated model of three-phase PWM rectifier based on onecycle control has been constructed in the saber software, according to the principle of one cycle-control and the rectifier. The core circuits of the control circuit of the three phase rectifier, such as the adder circuit, the comparison circuit, and the integrator circuit, and so on, are established in the saber software. The simulated circuit is shown in Fig.4.

The saber simulation software is one of the most powerful power electronic simulation software, and has the largest model library, and can satisfy the simulation requirement of the design that used in aircraft and power system. The simulation parameters of the three phase rectifier are listed as follows: the three-phase $\mathrm{AC}$ input voltage is $115 \mathrm{~V} / 400 \mathrm{~Hz}$; the output voltage of DC side is $300 \mathrm{~V}$; the output power is $6 \mathrm{KW}$; the boost inductor is $0.3 \mathrm{mH}$, the filter capacitor of DC side is $1000 \mathrm{u}$; the value $\mathrm{Kp}=46$, $\mathrm{Ki}=0.34$; and the switching frequency is $100 \mathrm{~K}$. The waveform of the input voltage and current of the rectifier is shown in Fig.5, and the output dc voltage waveform of the rectifier based on one-cycle control is shown in Fig.6. The simulation results is shown that the input current is identical with its input voltage, and the THD of input current is about $4.47 \%$ within the range of GJB181A for different loads. It is obvious that the rectifier can acquire the high power factor from the simulation results. The dc output voltage is $295 \mathrm{~V}$, and reaches to the requirement of design.

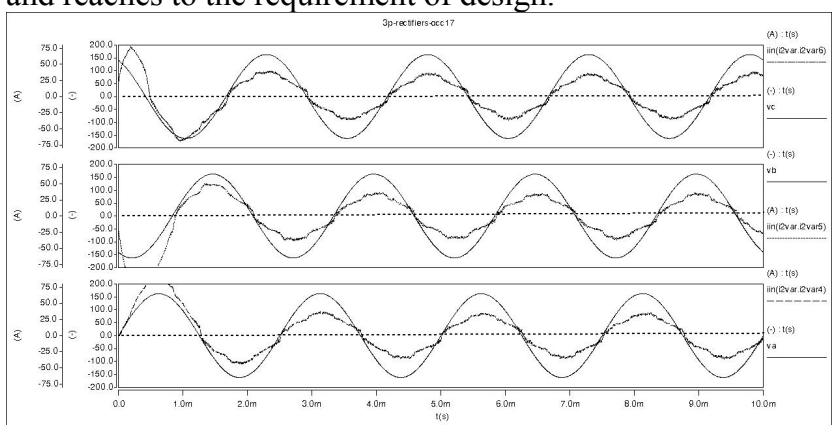

Figure 5. The waveforms of the input voltage and current

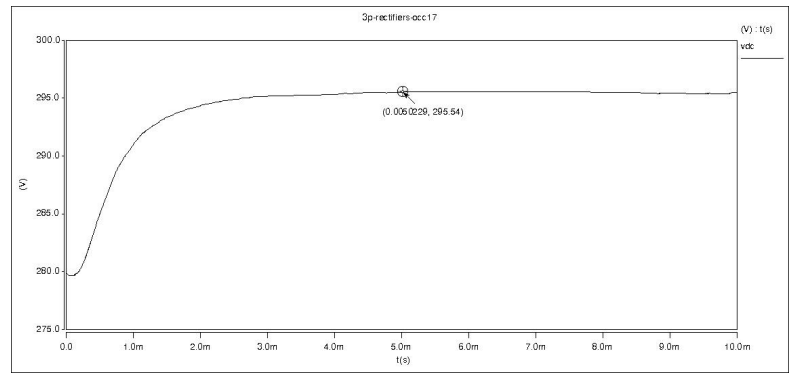

Figure 6. The waveforms of the output dc voltage

In order to verify the ability of the power with load, we increase the load, the simulated phase current and voltage waveforms of the three-phase PWM rectifier with high power is shown in Fig7. It is shown in Fig7, The waveforms of the input voltage and current is good while increasing the load. We can see that the waveforms of input voltage and current become worse through simulating.

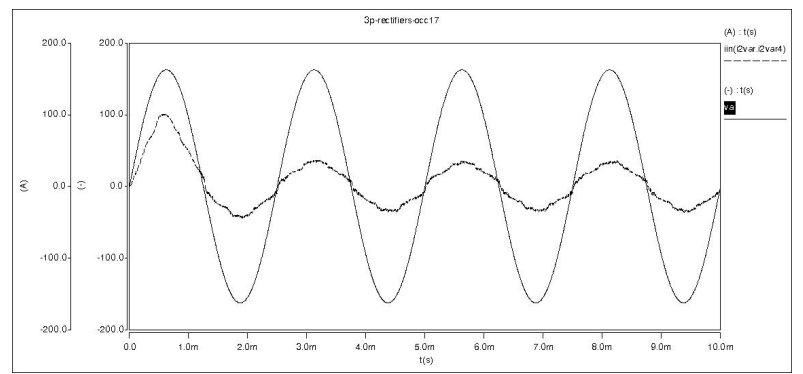

Figure 7. Simulated current waveforms of input voltage and current with bigger load

\section{CONCLUSION}

The research of the high power factor rectifier used in the aircraft is described in this paper. The simulation results show that the input current and the input voltage have the same phase, that is to say, the power factor of the rectifier close to unit power factor. The rectifier based on one-cycle control technology can achieve the high power factor. The output dc voltage basically reaches to the requirement of design. It is shown that the results of the three phase rectifier based on one-cycle control is very reliable and stable method.

\section{REFERENCES}

[1] ZHANG Housheng; HU Zhenying; LIU Xueting. Study of a General Three-Phase PFC Controller Based on One Cycle Control. TRANSACTIONS OF CHINA ELECTROTECHNICAL SOCIETY[J], 2004, PP:73-76.

[2] Qiao C; Taotao Jin; and K M. Smedley. One-Cycle Control of ThreePhase Active Power Filter With Vector Operation. IEEE TRANSACTIONS ON INDUSTRIAL ELECTRONICS, VOL. 51, NO. 2, APRIL 2004.

[3] Chongming Qiao,Keyue M Smedley.Three-phase unity-power-factor star-connected switch rectifier with unified constant-frequency integration control. IEEE Transactions on Power Electronics, 2003, PP:952 957.

[4] Yang Degang; Liu Runsheng and Zhao Liangbing. Current controller design of a three-phase high-power-factor rectifier, Transactions of China electrotechnical society, Vol.15, No.2, PP:83-87. 